\title{
A rare gastric neuroendocrine carcinoma coexisting with Brunner's gland adenoma: A case report
}

\author{
JIAN-MING WEI ${ }^{*}$, ZI-ZHEN ZHANG ${ }^{*}$, YAN-YING SHEN, DAN-PING SHEN and XING-ZHI NI \\ Department of General Surgery, Ren Ji Hospital, Shanghai Jiao Tong University School of Medicine, \\ Shanghai 200127, P.R. China
}

Received May 19, 2014; Accepted January 22, 2015

DOI: $10.3892 / \mathrm{ol} .2015 .3465$

\begin{abstract}
Gastric neuroendocrine carcinoma (G-NEC) is a rare neoplasm known for its aggressive behavior and poor prognosis. Brunner's gland adenoma (BGA) is a rare benign proliferative lesion that develops most commonly in the duodenum. To the best of our knowledge, no cases of G-NEC coexisting with BGA have previously been reported. The present study therefore reports the first case of G-NEC combined with BGA. A 67-year-old female presented with upper abdominal discomfort. No distant metastases were detected upon pre-operative abdominal computed tomography imaging. The patient underwent a radical distal gastrectomy, D2 lymphadenectomy and Billroth I gastroenterostomy. The resected masses were histologically confirmed to be G-NEC and BGA, respectively. The patient did not receive neoadjuvant or adjuvant chemotherapy or radiotherapy, and remains alive with no evidence of metastasis or recurrence at four years post-surgery.
\end{abstract}

\section{Introduction}

Gastric neuroendocrine carcinoma (G-NEC) is a malignant subtype of gastroenteral neuroendocrine tumor (GE-NET). It is a rare neoplasm known for its malignant biological behavior, neuroendocrine characteristics and poor prognosis (1). G-NECs account for $0.1-0.2 \%$ of all gastric malignancies; however, due to a lack of typical clinical presentation, it is difficult to diagnose prior to surgery (1). The incidence of G-NEC has been increasing each year and surgery is the only effective treatment option.

Correspondence to: Dr Xing-Zhi Ni, Department of General Surgery, Ren Ji Hospital, Shanghai Jiao Tong University School of Medicine, 1,630 Dongfang Road, Shanghai 200127, P.R. China

E-mail: georgeniwjc@163.com

*Contributed equally

Key words: gastric neuroendocrine carcinoma, Brunner's gland adenoma, prognosis
Duodenal Brunner's gland adenoma (BGA) is a rare, benign lesion that accounts for $10.6 \%$ of all benign duodenal tumors (2). The original description of BGA was provided by Salvioli (3) in 1876, and since then, only 200 cases have been reported in China (4). BGA is typically associated with no symptoms; however, in symptomatic patients, BGA presents with hemorrhagic or obstructive indicators of disease. The treatment of BGA varies according to the size of the tumor, the symptoms exhibited and the risk of malignancy (3). The pathogenesis of BGA is unclear, but malignant transformation to duodenal cancer has been reported in the literature (5). To the best of our knowledge, no cases of G-NEC coexisting with BGA have previously been reported. The present study therefore reports the first case of G-NEC combined with BGA. Written informed consent was obtained from the patient's family.

\section{Case report}

A 67-year-old female presented to a private hospital with a two-month history of vague abdominal discomfort, without nausea, vomiting, acid reflux or eructation. The patient had no significant past medical history, including no family history of cancer or surgery. An intraluminal mass measuring $2.0 \times 1.5 \times 1.1 \mathrm{~cm}$ was observed in the anterior wall of the duodenum. Esophagogastroduodenoscopy showed a large mucosal bulge measuring $5.0 \times 3.5 \times 1 \mathrm{~cm}$, with ulceration, in the antral lesser curvature of the stomach. The mass exhibited clear boundaries and a wide tumor pedicle. The patient was transferred to Ren Ji Hospital, Shanghai Jiao Tong University School of Medicine (Shanghai, China), due to frequent abdominal pain. Laboratory tests and tumor biomarker analysis for $\alpha$-fetoprotein, carcinoembryonic antigen, carbohydrate antigen 19-9 were normal. An abdominal plain computed tomography (CT) scan revealed an equal density shadow combined with uneven thickening. During three phases of intravenous contrast-enhanced CT, the lesions showed marked enhancement at the arterial phase and loss of enhancement at the venous and equilibrium phases. No liver metastases were detected (Fig. 1).

The patient underwent a radical distal gastrectomy, D2 lymphadenectomy and Billroth-I gastroenterostomy. Hematoxylin and eosin (HE) staining of the resected specimen revealed a mixture of atypical small and large G-NEC cells, with a 
A

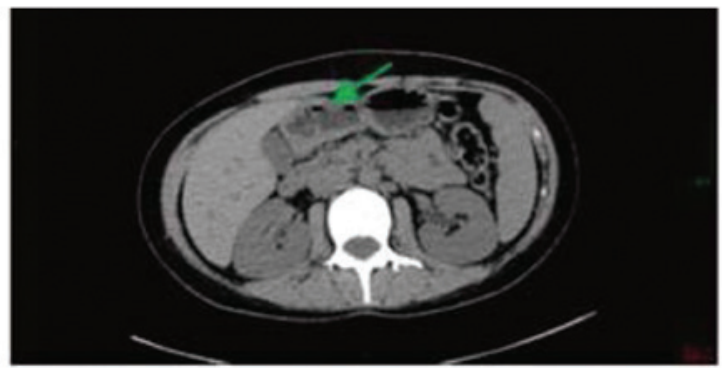

C

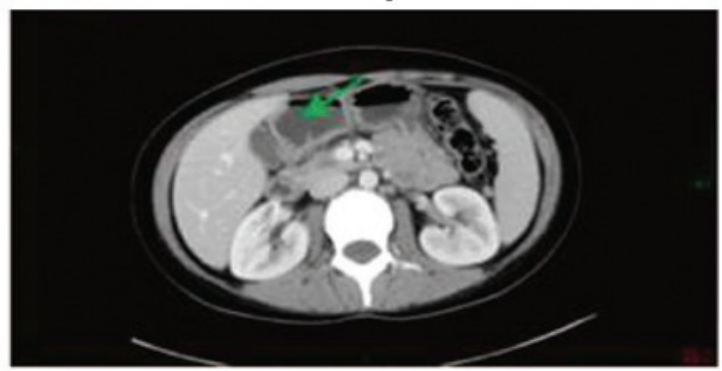

B

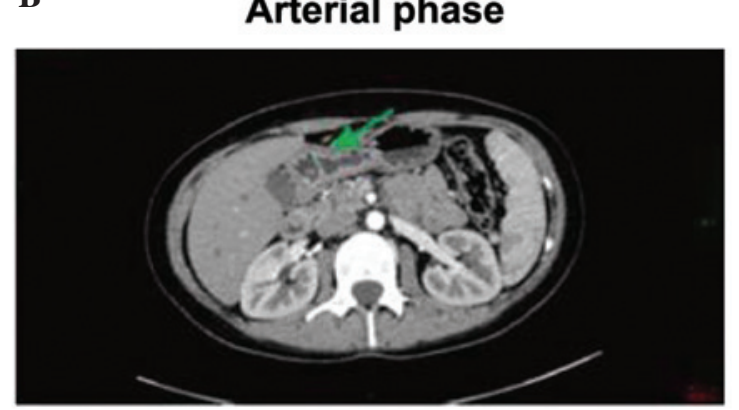

D

\section{Equilibrium phase}

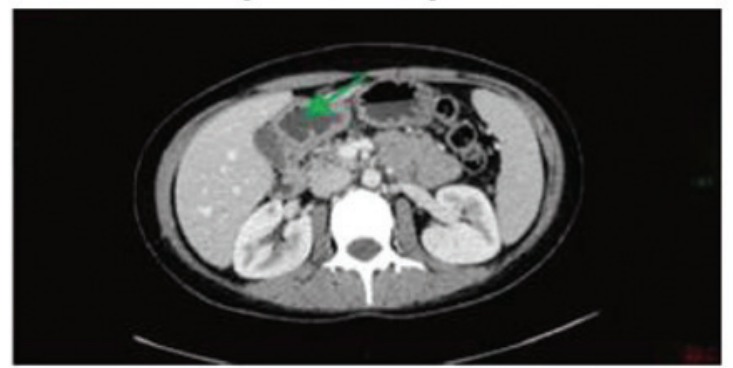

Figure 1. (A) Plain computed tomography scan showed an equal density shadow combined with uneven thickening. The lesions showed (B) marked enhancement at arterial phase and loss of enhancement at (C) venous and (D) equilibrium phases. No liver metastases were detected.
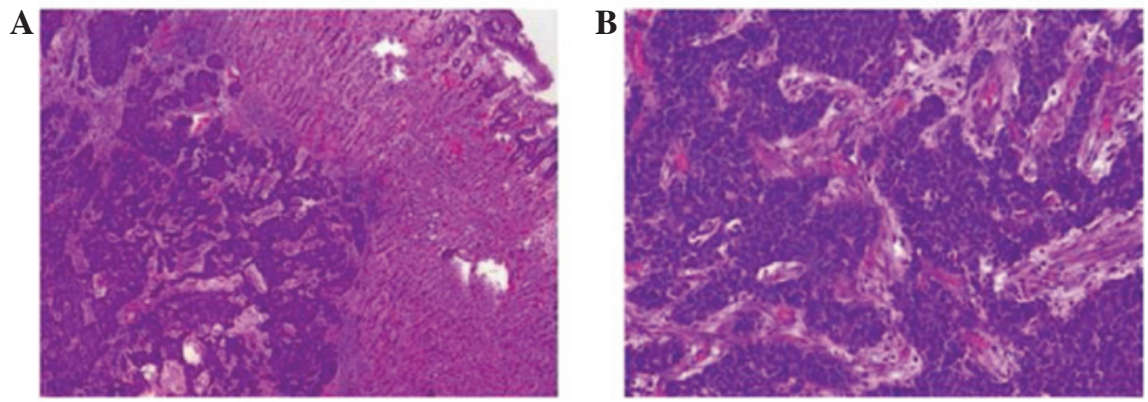

Figure 2. Microscopic examination of the hematoxylin and esoin-stained resected specimen revealing a mixture of atypical small and large cells, with a rose-like distribution, reduced cytoplasm and homogeneous fine nuclear chromatin. Mitotic figures were common. (A) x100 magnification. (B) x200 magnification.
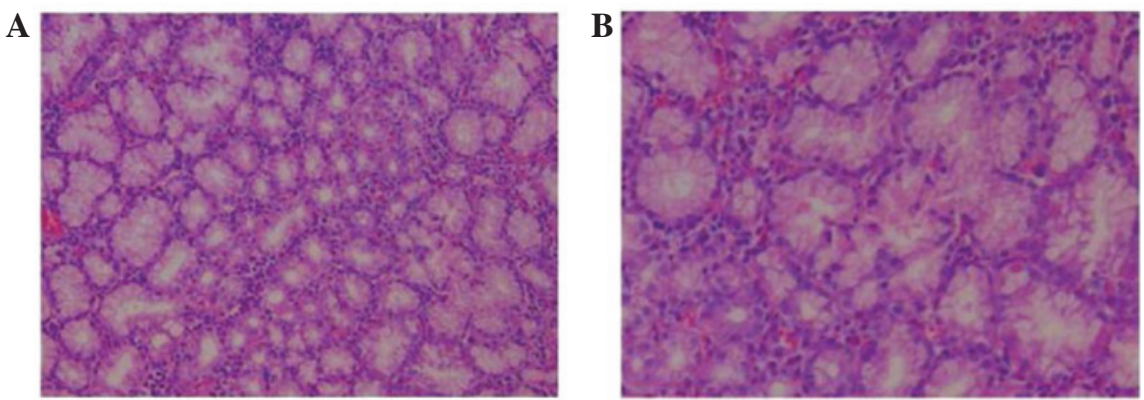

Figure 3. Microscopic examination of the hematoxylin and eosin-stained pedicle mass revealing that the lobules forming the tumor were composed of normal-appearing Brunner's glands with the usual mixture of normal tissues, including ducts, adipose tissue and lymphoid tissue. (A) x100 magnification (B) $\times 200$ magnification.

rose-like distribution, reduced cytoplasm and homogeneous fine nuclear chromatin (Fig. 2). Mitotic figures were common. Deep myometrial invasion and fuzzy boundaries were observed. One out of two lymph nodes in the lesser curvature of the stomach was positive for metastasis. The tumor was staged as T2N1M0, stage IIIB, according to the European Neuroendocrine Tumor Society guidelines (6). In addition, an intraluminal pedicle mass measuring $2.0 \times 1.5 \times 1.1 \mathrm{~cm}$ was observed in the anterior 

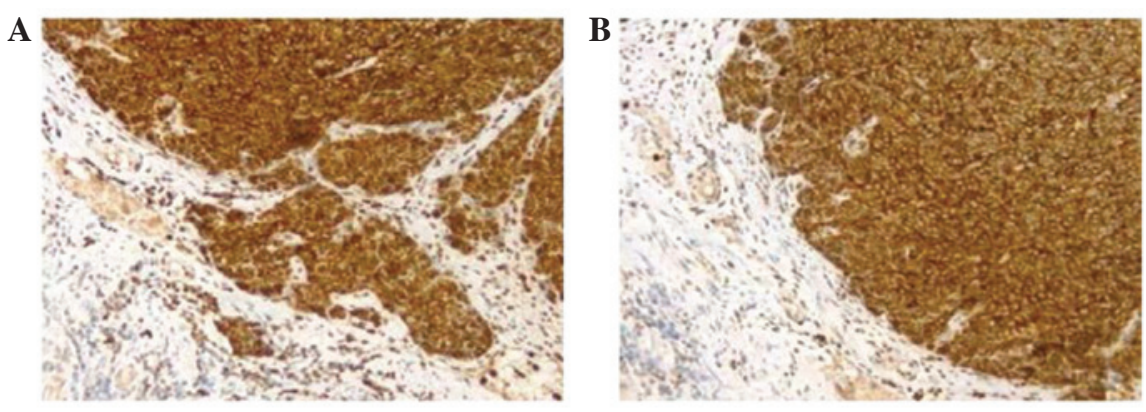

Figure 4. Gastric neuroendocrine carcinoma stained positively for (A) chromogranin (CgA) and (B) synaptophysin (Syn). Syn and CgA are present with a diffuse cytoplasmic distribution (x200 magnification).

wall of the duodenum, with a clear boundary and wide tumor pedicle. The surface of the mass was smooth, without ulceration or erosion, and was covered by intestinal mucosa. The tumor section was sallow, lobulated and soft. HE staining revealed that the tumor lobules were composed of normal-appearing Brunner's glands, with the usual mixture of normal tissues, including ducts, adipose tissue and lymphoid tissue. The observed gland hyperplasia with chronic inflammation was consistent with the diagnosis of BGA (Fig. 3).

Immunohistochemical analysis of the antral tumor specimen was performed following the surgery. In all tumours, immunostaining was classified as membranous, cytoplasmic, membranous and cytoplasmic (heterogeneous tissue labelling) or negative. Results were considered to be negative (-) when no immunostaining was detected, very weak $(+)$ when staining was present in $<20 \%$ of tumor cells, weak $(++)$ when staining was present in $20-50 \%$ of tumor cells, and strong $(+++)$ when staining was present in $>50 \%$ of tumor cells. Immunohistochemical analysis demonstrated positive staining for cytokeratin $(\mathrm{CK}) 7(+), \mathrm{CK} 8(+)$, cluster of differentiation 56(+), chromogranin $\mathrm{A}(\mathrm{CgA})(+), \mathrm{Ki}-67(+++)$ and $\mathrm{p} 53(+++)$. The tumor was negative for p63. Immunohistochemical staining for synaptophysin (Syn) and CgA were positive, and revealed diffuse cytoplasmic distribution for each protein (Fig. 4). These results are consistent with a diagnosis of G-NEC.

The patient did not receive radiotherapy or chemotherapy. Abdominal CT, magnetic resonance imaging and endoscopy were performed every six months for the first two years after surgery. For the next two years, abdominal CT and endoscopy were performed every 12 months. No metastatic lesions were found during the four-year follow-up period.

\section{Discussion}

Gastrointestinal neuroendocrine tumors, also referred to as GE-NETs, are a rare low-grade malignancy, accounting for $\sim 2 \%$ of all gastrointestinal malignancies (7). The incidence of GE-NETs has been increasing in recent years. Currently, there are no uniform grading standards for GE-NETs. According to the latest classification defined by the World Health Organization in 2010, neuroendocrine tumors are classified into three grades based on the Ki-67 labeling index (8). Grade 1 tumors have a $\mathrm{Ki}-67$ labeling index of $\leq 4 \%$, grade 2 tumors have an index of $3-20 \%$ and grade 3 tumors have an index of $\geq 20 \%$. G-NECs, the more malignant subtype of GE-NETs, account for $0.1-0.2 \%$ of all gastric malignancies (9). Immunohistochemical analysis of the tumor from the present patient revealed a Ki-67 labeling index of $67 \%$, resulting in a diagnosis of grade 3 G-NEC.

G-NEC exhibit no specific early clinical manifestations, while the late clinical manifestations include upper abdominal pain and progressive dysphagia. A few cases of G-NEC have been reported with blood in the stool and anemia (10); thus, the tumors may be confused with gastric adenocarcinoma or gastric lymphoma. Indeed, the present patient was misdiagnosed with gastric cancer prior to surgery. The diagnosis of G-NEC relies on the morphological characteristics of the tumor and immunohistochemical analysis. G-NECs secrete the neural markers Syn and CgA. Any patient with positive expression of these markers can be diagnosed with a G-NEC (11).

Surgery is required for patients diagnosed with G-NEC (12). Other treatment options include biological therapy, molecular targeted therapy, chemotherapy and radiation therapy. Novel biological and targeted therapies have been the focus of G-NEC treatment in recent years. Biological therapy has focused on somatostatin (SST), which specifically binds the SST receptor (SSTR) expressed on the surface of NET cells, thereby inhibiting the secretion of various bioactive substances, including 5-hydroxytryptophan, insulin and gastrin (13), resulting in the improvement of clinical symptoms. SST suppresses tumor growth by blocking cells in the $\mathrm{G}_{1}$ phase of the cell cycle, regulating immunity through an SSTR independent mechanism, inhibiting angiogenesis and promoting apoptosis (14). It has been reported that large doses of SST analogs can lead to tumor cell apoptosis and inhibit tumor growth, but these results remain controversial (15). Tyrosine kinase inhibitors are the most commonly used agents in targeted therapy and the mammalian target of rapamycin (mTOR) receptor has been the focus of several studies. In the phase III RADIANT-3 clinical trial, everolimus, an inhibitor of the PI3K/AKT/mTOR signaling pathway, significantly extending the time to progression for patients in the drug arm compared with those in the placebo arm of the study. The patient in the present study did not receive biological treatment prior to or following surgery, as no symptoms developed that were associated with carcinoid syndrome. Additionally, no post-operative chemotherapy was administered. The patient remains alive and free of symptoms of carcinoid syndrome at four years post-surgery.

BGA is usually asymptomatic, but may exhibit clinical manifestations that can range from non-specific symptoms to gastrointestinal bleeding or obstruction only detectable by an upper gastrointestinal endoscopy $(16,17)$. It has been suggested that BGA is not a true tumor, but a lesion of nodular 
hyperplasia or hamartoma (18). BGA generally arises in individuals aged 50-60 years. The tumor is often small, ranging in size from 1-10 cm, with an average size of $1-2 \mathrm{~cm}$. At present, the etiology of BGA is not clear, but it is believed to be caused by duodenal inflammation or hyperacidity, leading to hyperplasia of Brunner's glands and hamartoma (19).

In conclusion, BGA is a rare benign lesion which may be diagnosed by histopathology. BGA has a good prognosis, however, we hypothesize that lesions must be resected if their shape or size changes significantly. In the present patient, BGA was discovered during the surgery for G-NEC. Further investigation is required to determine if the pathogenesis of BGA in this patient was associated with the neuroendocrine characteristics of the G-NEC or if it was just a coincidence.

\section{References}

1. Nishikura K, Watanabe H, Iwafuchi M, Fujiwara T, Kojima K and Ajioka Y: Carcinogenesis of gastric endocrine cell carcinoma: analysis of histopathology and p53 gene alteration. Gastric Cancer 6: 203-209, 2003.

2. Gao YP, Zhu JS and Zheng WJ: Brunner's gland adenoma of duodenum: a case report and literature review. World J Gastroenterol 10: 2616-2617, 2004.

3. Coskun A and Erkan N: Giant Brunner's gland adenoma as an unusual cause of anaemia: Report of a case. Radiol Oncol 45: 129-131, 2011.

4. Stewart ZA, Hruban RH, Fishman EF and Wolfgang CL: Surgical management of giant Brunner's gland hamartoma: case report and literature review. World J Surg Oncol 7: 68, 2009.

5. Masaru Koizumi, Sata N, Yoshizawa K, Kurihara K and Yasuda Y: Carcinoma arising from Brunner's gland in the duodenum after 17 years of observation - a case report and literature review. Case Rep Gastroenterol 1: 103-109, 2007.

6. Rindi G, Klöppel G, Alhman H, et al; all other Frascati Consensus Conference participants; European Neuroendocrine Tumor Society (ENETS): TNM staging of foregut (neuro)endocrine tumors: a consensus proposal including a grading system. Virchows Arch 449: 395-401, 2006.
7. Toumpanakis CG and Caplin ME: Molecular genetics of gastroenteropancreatic neuroendocrine tumors. Am J Gastroenterol 103: 729-732, 2008.

8. Rindi G, Arnold R, Bosman FT, Capella C, Klimstra DS, Klöppel G, et al: Nomenclature and classification of neuroendocrine neoplasms of the digestive system. In: WHO Classification of Tumors of the Digestive System. Bosman FT, Carneiro F, Hruban RH and Theise ND (eds). 4th edition. IARC Press, Lyon, France, pp13-14, 2010.

9. Uchiyama C, Tamura S, Nakatsuka S, et al: Immunohistochemical consistency between primary tumors and lymph node metastases of gastric neuroendocrine carcinoma. World J Surg Oncol 10: $115,2012$.

10. Massironi S, Sciola V, Peracchi M, et al: Neuroendocrine tumors of the gastro-entero-pancreatic system. World J Gastroenterol 14: 5377-5384, 2008.

11. Solcia E, Klöppel G and Sobin LH: WHO International Histological Classification of Tumors: Histological Typing of Endocrine Tumors. 2nd edition. Springer-Verlag, Berlin, pp56-70, 2000.

12. Fendrich V and Bartsch DK: Surgical treatment of gastrointestinal neuroendocrine tumors. Langenbecks Arch Surg 396: 299-311, 2011.

13. Susini $C$ and Buscail L: Rationale for the use of somatostatin analogs as antitumor agents. Ann Oncol 17: 1733-1742, 2006.

14. Mejias M, Garcia-Pras E, Tiani C, et al: The somatostatin analogue octreotide inhibits angiogenesis in the earliest, but not in advanced, stages of portal hypertension in rats. J Cell Mol Med 12: 1690-1699, 2008

15. Panzuto F, Di Fonzo M, Iannicelli E, et al: Long term clinical outcome of somatostatin analogues for treatment of progressive, metastatic, well-differentiated entero-pancreatic endocrine carcinoma. Ann Oncol 17: 461-466, 2006.

16. Matsumoto T, Iida M, Matsui T, et al: A large Brunner's gland adenoma removed by endoscopic polypectomy. Endoscopy 22: 192-193, 1990.

17. Rocco A, Borriello P, Compare D, De Colibus P, Pica L, Iacono A and Nardone G: Large Brunner's gland adenoma: case report and literature review. World J Gastroenterol 12: 1966-1968, 2006.

18. Rufenacht H, Kasper M, Heitz PU, et al: 'Brunneroma': hamartoma or tumor?. Pathol Res Pract 181: 107-111, 1986.

19. Levine JA, Burgart LJ, Batts KP and Wang KK: Brunner's gland hamartomas: clinical presentation and pathological features of 27 cases. Am J Gastroenterol 90: 290-294, 1995. 